\title{
Gastrointestinale Stromatumoren Adjuvante Therapie mit Imatinib senkt die Rezidivhäufigkeit
}

\begin{abstract}
DeMatteo RP, Ballman $\mathrm{KV}$, Antonescu CR et al. Adjuvant imatinib mesylate after resection of localised, primary gastrointestinal stromal tumour; a randomised, double-blind, placebo-controlled Trial. Lancet 2009;373:1097-104.
\end{abstract}

\begin{abstract}
Hintergrund und Fragestellung
Gastrointestinale Stromatumoren (GIST) sind potenziell maligne Tumoren, die früher auch als Leiomyome oder Leiomyosarkome des Gastrointestinaltrakts bezeichnet wurden. Charakteristisch ist die Expression von CD117, dem Typ-III-Tyrosinkinaserezeptor, KIT. Bei operablen Tumoren ist die Therapie der Wahl die chirurgische Resektion. Leider entwickeln bis zu 50\% der Patienten auch nach R0-Resektion innerhalb von fünf Jahren ein Rezidiv bzw. Metastasen. Dabei ist das Rezidivrisiko umso höher, je größer der Tumor und je höher die Mitoserate des Tumors ist [1].

Aufgrund der mangelnden Wirksamkeit gängiger Chemotherapeutika war bislang keine adjuvante Therapie etabliert. Seit mehreren Jahren ist der Tyrosinkinaseinhibitor Imatinib zur Behandlung der metastasierten GIST zugelassen.

Ziel der vorliegenden Studie war der Nachweis eines verbesserten rezidivfreien Überlebens durch eine adjuvante Therapie mit Imatinib nach der Resektion eines primären GIST.
\end{abstract}

\section{Patienten und Methodik}

In die randomisierte, doppelblinde, placebokontrollierte Multicenterstudie wurden im Verlauf von fünf Jahren über 700 Patienten eingeschlossen. Sie erhielten nach R0-Resektion eines GIST für die Dauer eines Jahres entweder täglich $400 \mathrm{mg}$ Imatinib oder Placebo. Primärer Studienendpunkt war das rezidivfreie Überleben.

\section{Ergebnisse}

Im Behandlungsarm traten signifikant weniger Rezidive als im Placeboarm auf (Abb. 1). Der positive Effekt der adjuvanten Therapie korrelierte mit der Tumorgröße. Bei Patienten mit Tumoren $>10$ cm konnte das Rezidivrisiko nahezu halbiert werden, während für Patienten mit kleinen Tumoren kaum ein Nutzen erkennbar war. Unabhängig von der Tumorgröße hatte die adjuvante Therapie jedoch keinen Einfluss auf das Gesamtüberleben.

Schlüsselwörter: Gastrointestinale Stromatumoren - adjuvante Therapie - Imatinib

\section{Die Therapie primär operabler GIST hat sich gewandelt Kommentar von Natascha Nüssler}

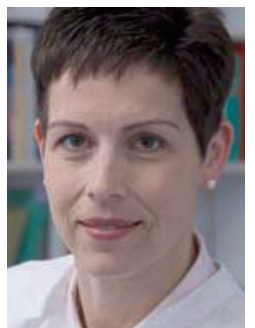

Prof. Dr. med. Natascha Nüssler Chefärztin der Klinik für Allgemein- und Viszeralchirurgie am Klinikum Neuperlach,

München. E-Mail:

natascha.nuessler@ klinikum-muenchen.de
D e adjuvante Therapie mit Imatinib kann die Rezidivhäufigkeit nach Resektion eines primären GIST senken. Da vor allem Patienten mit einem hohen Rezidivrisiko von der Therapie profitieren, ist eine Risikostratifizierung nötig. DeMatteo et al. haben hierfür lediglich die Tumorgröße verwendet. Prognostisch relevant sind aber auch die Proliferationsrate des Tumors, die unterschiedlichen Mutationen des KIT-Rezeptors sowie die Tumorlokalisation. Diese Parameter sollten künftig in die Risikoanalyse miteinbezogen werden. Unklar bleibt die Dauer der adjuvanten Therapie. Im Behandlungsarm traten Rezidive gehäuft im zweiten Jahr nach der Operation auf. Es ist daher fraglich, ob eine einjährige adjuvante Therapie ausreicht.

In dieser Studie nicht thematisiert, aber von großem klinischem Interesse ist die Resistenzentwicklung der GIST [2, 3]. Aufgrund der relativ kurzen Nachbeobachtungszeit und des Cross-over-
Studiendesigns (Patienten des Placeboarms durften bei Rezidiv zu Imatinib wechseln) war es nicht überraschend, dass keine Unterschiede im Gesamtüberleben beobachtet werden konnten.

\section{Fazit}

Die Therapie primär operabler GIST hat sich gewandelt: Bei c-KIT-positivem GIST und hohem Rezidivrisiko sollte nach der Tumorresektion eine adjuvante Behandlung mit Imatinib erfolgen. Unklar bleibt die Dauer dieser Therapie.

\section{Literatur}

1. Casali PG et al. Ann Oncol 2008 May;19 Suppl 2:ii35-8.

2. Antonescu CR et al. Clin Cancer Res 2005;11:4182-90.

3. Gajiwala KS et al. Proc Natl Acad Sci USA 2009;106: 1542-47.

[Erstmals publiziert in Gastro News 2009;1:18.] 